\title{
Influence of atmospheric stratification on the integral scale and fractal dimension of turbulent flows
}

\author{
Manuel Tijera $^{1}$, Gregorio Maqueda ${ }^{2}$, and Carlos Yagüe $^{3}$ \\ ${ }^{1}$ Applied Mathematics Dpt. (Biomathematics), Complutense University of Madrid, Madrid, Spain \\ ${ }^{2}$ Astronomy, Astrophysics and Atmospheric Science Dpt., Complutense University of Madrid, \\ Madrid, Spain \\ ${ }^{3}$ Geophysics and Meteorology Dpt., Complutense University of Madrid, Madrid, Spain
}

Correspondence to: Manuel Tijera (mtijera@fis.ucm.es)

Received: 21 December 2015 - Published in Nonlin. Processes Geophys. Discuss.: 11 February 2016 Revised: 8 September 2016 - Accepted: 19 September 2016 - Published: 14 November 2016

\begin{abstract}
In this work the relation between integral scale and fractal dimension and the type of stratification in fully developed turbulence is analyzed. The integral scale corresponds to that in which energy from larger scales is incoming into a turbulent regime. One of the aims of this study is the understanding of the relation between the integral scale and the bulk Richardson number, which is one of the most widely used indicators of stability close to the ground in atmospheric studies. This parameter will allow us to verify the influence of the degree of stratification over the integral scale of the turbulent flows in the atmospheric boundary layer (ABL). The influence of the diurnal and night cycles on the relationship between the fractal dimension and integral scale is also analyzed. The fractal dimension of wind components is a turbulent flow characteristic, as has been shown in previous works, where its relation to stability was highlighted. Fractal dimension and integral scale of the horizontal $\left(u^{\prime}\right)$ and vertical $\left(w^{\prime}\right)$ velocity fluctuations have been calculated using the mean wind direction as a framework. The scales are obtained using sonic anemometer data from three elevations 5.8, 13 and $32 \mathrm{~m}$ above the ground measured during the SABLES 98 field campaign (Cuxart et al., 2000). In order to estimate the integral scales, a method that combines the normalized autocorrelation function and the best Gaussian fit $\left(R^{2} \geq 0.70\right)$ has been developed. Finally, by comparing, at the same height, the scales of $u^{\prime}$ and $w^{\prime}$ velocity components, it is found that the turbulent flows are almost always anisotropic.
\end{abstract}

\section{Introduction}

The aim of this paper is to investigate the possible correlations between the integral scale of the turbulent stratified flows in the atmospheric boundary layer and parameters characterizing topological features of the wind velocity field, such as fractal dimension and its stability properties, studied through the bulk Richardson number. We are aware that there is a lack of investigations between the integral scale and the fractal dimension. The size of the integral scale of the horizontal and vertical components and fractal dimension of wind velocity near the earth's surface in the boundary layer are determined. Also, these magnitudes are compared between them and vs. other parameters such as the bulk Richardson number. It is assumed that the turbulence is the primary agent that causes changes in the boundary layer. In turbulent flows it is observed that time series of meteorological variables such as wind velocity, temperature, pressure and other atmospheric mechanical magnitudes fluctuate in a disordered way, with peaks extremely sharp and irregular in space and time variations. The complicated nature of these series indicates that the motion of the air is turbulent. If we take a good look at the variety of fluctuations of different periods and amplitudes observed in them, we could explain the complicated structure of turbulence. The irregularity of the time series obeys the existence of different sizes and timescales and also the nonlinear transfer of energy that exists between them in the turbulent flows (Monin and Yaglom, 1971)

The irregular behavior of these flows is also due to waves and turbulence that are often superimposed onto a mean 
wind (Stull, 1988). If we filter the mean wind and waves in the appropriate range, we will only have turbulence. Some previous works present results of this procedure (Tijera et al., 2008). In this work, the series of wind velocities in the three directions $x, y$ and $z$ recorded by the anemometer are divided into series of non-overlapping 5 min length. Each of these series applies the necessary rotations to get the $x$ axis in the mean wind direction (mean $v$ is zero) and zero mean vertical velocity ( $w$ vertical component) (Kaimal and Finnigan, 1994). We filter horizontal and vertical mean wind velocity, obtaining the time series of fluctuations of the velocity in both directions $\left(u^{\prime}=u-\bar{u}, w^{\prime}=w-\bar{w}\right)$.

When we observe these time series such as wind velocity, they vary in an irregular shape, and in spite of their complexity present a self-similarity structure (Frisch, 1995). This is a common property of the fractals, so that wind velocity could be considered a fractal magnitude. The modern physical notion of fractals is largely known due to Mandelbrot (1977, 1985), but the mathematical notion of curves lines or sets having noninteger dimensions is much older (Hausdorff, 1918; Besicovitch, 1929). An analysis that compares the Haussdorff dimension and Kolmogorov capacities of selfsimilar structures with noninteger fractal dimensions (Kolmogorov capacity or box counting dimension) was presented by Vassilicos (Vassilicos and Hunt, 1991). The wind velocity vs. time are irregular curves of this type, with noninteger dimensions. These values correspond to the fractal dimension. A way of measuring the complexity of these series is by means of fractal dimensions. The fractal dimension of wind components is a characteristic of turbulent flow, as has been shown in previous works where its relation to stability was highlighted (Tijera et al., 2012).

In this paper the integral scales of $u^{\prime}$ and $w^{\prime}$ components are compared. The scales are calculated using sonic anemometer data from three elevations $5.8(\sim 6), 13$ and $32 \mathrm{~m}$ above the ground at the main tower site of the SABLES 98 field campaign. Turbulent motion of the atmospheric flows occurs through a broad range of scales, from the smallest ones that are usually defined as the scales at which the motion dissipates into heat due to the viscosity of the fluid, to the larger scales corresponding to the integral scale. The integral scale can be defined in several ways: the larger scale of the flow, or the scale above which the Fourier transform has a slope inferior to $a-5 / 3$ slope, such as where the turbulent kinetic energy (TKE) is maximum. Micrometeorological studies have found integral scales varying in a huge range, from around 100 to $1000 \mathrm{~m}$ (Teunissen, 1980; Kaimal and Finnigan, 1994).

We study the anisotropy of the turbulent atmospheric flows in these scales by comparing the integral scale of fluctuations of the velocity component along the mean wind direction and the vertical component at three different levels above the ground $(5.8,13.5,32 \mathrm{~m})$.

\section{Theoretical background}

The irregular behavior of the atmospheric turbulent fluxes in the boundary layer at large Reynolds numbers leads us to be interested in calculating their fractal dimension. Fractal dimension could help us to classify the irregularity of these flows. The more irregular the flow, the greater its fractal dimension. Turbulent flows are characterized by the formation of many eddies of different length scales. Theses irregularities are due to the superimposition of eddies of different sizes, and this is related to a broad range of scales that exist in turbulence. These scales vary from the smallest scales as dissipative scales to larger scales as integral scales. This paper is concerned with the analysis of the relationship between the integral scale and the fractal dimension, as well as with the relationship between the integral scale with the bulk Richardson number, which provides a measure of the degree of stability in the flow, and how this turbulent flow is prone to developing instabilities. It is also used as a criterion for the existence or non-existence of turbulence in a stably stratified environment (a large positive value over a critical threshold is indicative of a decaying turbulence or a complete non-turbulence) (Arya, 2001).

In this section we describe the methodology applied to calculate the fractal dimension and the integral scale. The estimation of the fractal dimension of time series has been the most commonly used criterion to measure their chaotic structure; there exist different works in that direction (Grassberger and Procaccia, 1983; Shirer et al., 1997). One of the methods most commonly used to estimate the fractal dimension of atmospheric flows has been the mean slope method through the box-counting dimension using mean slopes of the graph of $\ln N(L)$ vs. $\ln (L)$ for small ranges of $L$, where $N(L)$ is the number of boxes of side $L$ necessary to cover the different points that have been registered in the physical space (velocity-time) (Falconer, 2000; Peitgen et al., 2004). As $L \rightarrow 0$, then $N(L)$ increases, and $N$ meets the following relation:

$N(L) \cong k L^{-d}$.

The value $d$ is the box-counting dimension that is an approximation of the Hausdorff dimension and is calculated approximately by means of least-square fitting of the representation of $\log N(L)$ vs. $\log L$, obtaining the straight line regression given by the following equation:

$\log N(L)=\log k-d \log L$.

The fractal dimension $d$ will be given by the slope of this equation as shown in Fig. 1.

In this paper we focus on calculating the integral scales for horizontal and vertical component fluctuations $u^{\prime}$ and $w^{\prime}$, and we studied their variations with respect to the fractal dimension and the bulk Richardson number, a turbulent parameter of stability. 


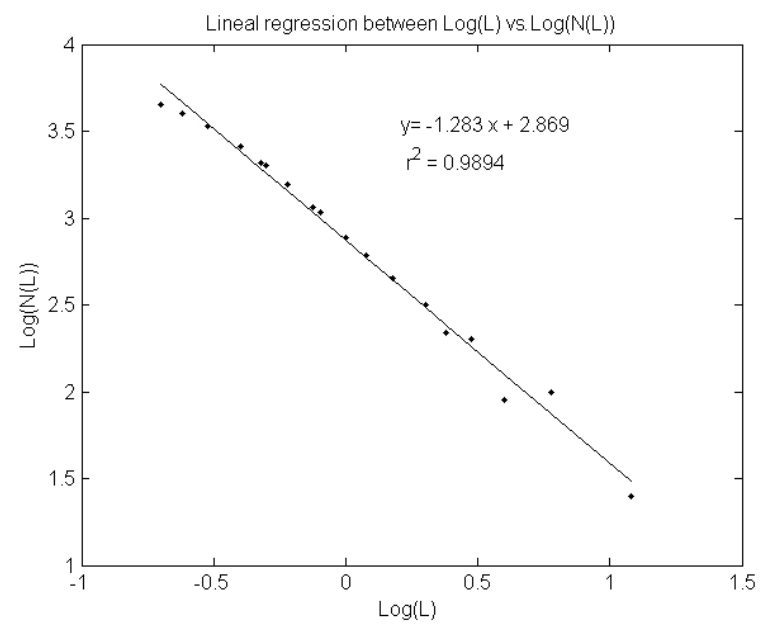

Figure 1. Example of linear regression between the number of nonempty boxes and the length side of the box. The slope $(d)$ is the fractal dimension of the $w^{\prime}$ component, $d=1.28 \pm 0.03$, for an example of the $w^{\prime}$ component of the wind velocity.

These integral scales have been estimated using the normalized autocorrelation function and a Gaussian fit. The velocity autocorrelation function as a function of $\tau$ (lag number) for the $u^{\prime}$ component is

$R(\tau)=\frac{\overline{u^{\prime}(t) u^{\prime}(t+\tau)}}{\overline{u^{\prime 2}(t)}}$.

The integral timescale is

$T_{L}=\int_{0}^{\infty} R(\tau) d \tau \approx \int_{0}^{\tau^{\prime}} R(\tau) d \tau$.

The integral timescale provides a measure of the scales of eddies in the $x$ direction of a flow field. In Eq. (2) we observed that $\tau^{\prime}$ denotes the last lag in the data series. In boundary layer observations this timescale can be related to a length by multiplying the mean wind velocity by the timescale. This requires the assumption of frozen turbulence known as Taylor's hypothesis (Panofsky and Dutton, 1984). The integral length scale can be defined as

$\lambda=\bar{v} T_{L}$.

The used method is based on a Gaussian fit of the normalized autocorrelation function $R(\tau)$ and we calculated the value of $\tau$ that verifies the following equation:

$\tau-\int_{0}^{\tau} R(\tau) d \tau=\int_{\tau}^{\tau^{\prime}} R(\tau) d \tau$.

Fig. 2 shows the Gaussian fit for an example of a data series of wind velocities with $\tau$ that verifies Eq. (6). This value allows us to calculate the integral timescale by multiplying it by the time interval between each lag.

\section{Description of data}

The data set was recorded at the Research Centre for the lower Atmosphere (CIBA in the Spanish acronym), located in Valladolid province (Spain), and was measured in the SABLES 98 experimental campaign. This research center was set up primarily to study the atmospheric boundary layer. The campaign took place from 10 to 27 September 1998 (Cuxart et al., 2000). This experimental site is a quite flat and homogeneous one that forms a high plain of nearly $200 \mathrm{~km}^{2}$, surrounded by crop fields and some small bushes strewn over the ground. The Duero River flows along the southeastern border of the high plain. The synoptic conditions during the period of study of 8 consecutive days (from 14 to 21 September) were controlled by a high-pressure terrain system that produces thermal convection during the diurnal hours and moderate to strong stable stratification during the nights.

In this work, data from sonic anemometers measured at a sampling rate of $20 \mathrm{~Hz}$ installed at $5.8(\sim 6), 13$ and $32 \mathrm{~m}$ are analyzed; $5 \mathrm{~min}$ non-overlapping series are used to evaluate the different parameters. At a rate of 20 data points per second, sonic anemometers can resolve integral scales between about 10 and $2000 \mathrm{~m}$ of the $u^{\prime}$ horizontal component and between 1 and $1000 \mathrm{~m}$ of the $w^{\prime}$ vertical component, depending on the height at which the sonic anemometer is positioned and at the wind speeds typically measured in the SABLES 98 experiment. We detect vertical scales over a broad range of scales from 1 to $1000 \mathrm{~m}$. The integral scales here are calculated based on the autocorrelation function, the mean wind velocity and integral timescale, and each of them can be expected to vary significantly. As the integral scales are the larger scales of turbulent flows, it is possible to detect vertical scales larger than heights at which the sonic anemometer is located.

\section{Results}

\subsection{Fractal dimension, integral scale and stability of stratification.}

In this paper we analyze the influence of stability of stratification on fractal dimension and integral scale. Different atmospheric surface-layer data are separated into thermal and dynamics stability classes based on a dimensionless parameter such as the bulk Richardson number $\mathrm{Ri}_{\mathrm{B}}$. This parameter represents the ratio of the production or destruction of turbulence by buoyancy and by wind shear strain that is caused by mechanical forces in the atmosphere:

$\mathrm{Ri}_{\mathrm{B}}=\frac{g}{\bar{\theta}} \frac{\Delta \bar{\theta} \Delta z}{(\Delta \bar{u})^{2}}$

where $g$ is the gravity acceleration and $\bar{\theta}$ the average potential temperature at the reference level; the term $\frac{g}{\bar{\theta}}$ is known 


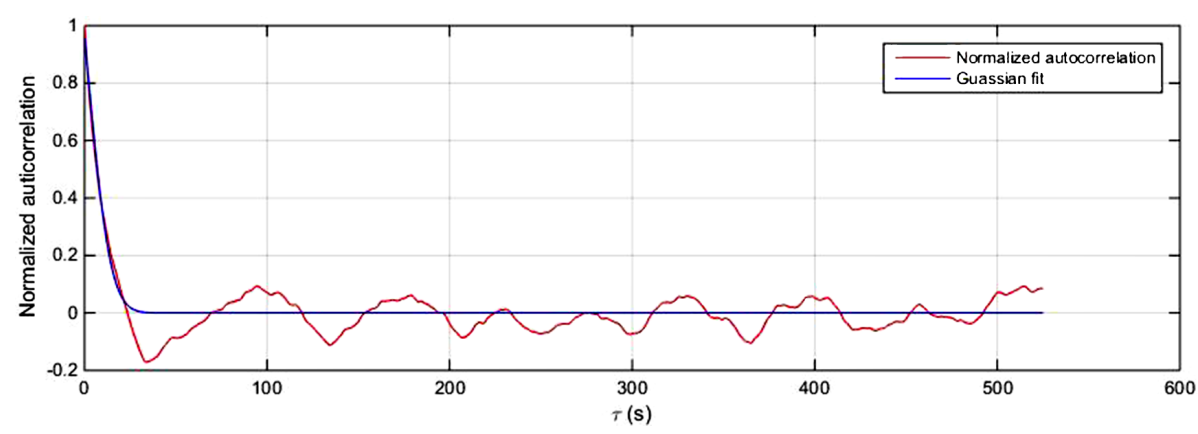

Figure 2. Gaussian fit for a data series of wind velocities $u^{\prime}$ component that allows us to calculate the integral scale.

as the buoyancy parameter. $\mathrm{Ri}_{\mathrm{B}}$ is positive for stable stratification, negative for unstable stratification and approximately zero for neutral stratification (Arya, 2001). The way to calculate this number is described next.

1. Calculation of the mean potential temperatures at height $z=32 \mathrm{~m}$, and close to the surface at $z=5.8 \mathrm{~m}$, namely $\overline{\theta_{32}}$ and $\overline{\theta_{5.8}}$, respectively, since $\Delta \bar{\theta}=\overline{\theta_{32}}-\overline{\theta_{5.8}}$. The potential temperature has been estimated as relative to ground level by using the following formula: $\Delta \theta=$ $\Delta T+\Gamma \Delta z, \Gamma=0.0098 \mathrm{~K} \mathrm{~m}^{-1}$ (Arya, 2001).

2. Obtaining of $\overline{u_{z}}$ by the mean wind velocity module at heights $z=32 \mathrm{~m}$ and $z=5.8 \mathrm{~m}$, denoted by $\overline{u_{32}}$ and $\overline{u_{5.8}}$, respectively, where $\Delta \bar{u}=\overline{u_{32}}-\overline{u_{5.8}}$.

Once the values of $\Delta \bar{\theta}, \Delta \bar{u}$ and $\Delta z$ have been obtained by means of Eq. (7), we calculate the bulk Richardson number in the layer between 32 and $5.8 \mathrm{~m}$.

In Fig. 3 we present the variation of the fractal dimension of the $u^{\prime}$ horizontal component of the velocity fluctuations along time at the three considered heights: 5.8,13 and $32 \mathrm{~m}$. The behavior of these variations is similar at the three heights. The $w^{\prime}$ component fluctuation presents an analogous behavior. The fractal dimension values are in a range between 1.30 and nearly 1.00 . We have found that during the diurnal hours the fractal dimension is bigger than at night (Tijera, 2012). We have no theoretical reason to explain this result, but a possible explanation of why this happens could be that fractal dimension is related to atmospheric stability and to the intensity of turbulence. It is well known that the intensity of turbulence grows as solar radiation increases, producing instability close to the ground, mainly in the noon hours. Therefore, one of the possible reasons for the increase in fractal dimension (FD) is the instability of the turbulent flow. On the other hand, during the nights a strong atmospheric stability usually exists, so the fractal dimension is usually smaller than during the diurnal hours.

In Fig. 4 it can be seen how the integral scale varies vs. time at the three heights. There are some questions that have not been clarified yet in the literature. For example: how do the diurnal and night cycles influence the integral scale?

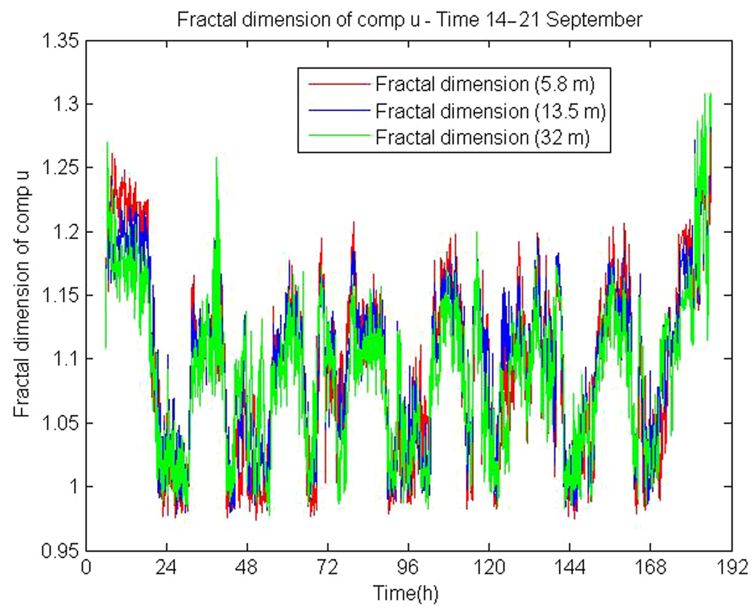

Figure 3. Variation of the fractal dimension vs. time for the $u^{\prime}$ component fluctuation at the three heights, showing the influence of the diurnal cycle.

Which is the mechanism responsible for the growth of this integral scale? It has been observed in previous works that under certain conditions the turbulent flows self-organize and develop large-scale structures that take place through an inverse cascade that occurs in stably stratified anisotropic flows (with or without rotation) (Smith and Waleffe, 2002; Marino et al., 2014). The inverse cascade mechanism might also be responsible for the growth of the integral scale in the stratified atmosphere. It is a fundamental issue that should be clarified in future research. As is indicated in Fig. 4, the integral scale for the $u^{\prime}$ component varies between around $100 \mathrm{~m}$ on their smaller scales and above $1500 \mathrm{~m}$ for their larger scales. The integral scales for the $w^{\prime}$ component are slightly lower than for the $u^{\prime}$ component. It is shown that these vertical scales can reach sizes of between a few tens of meters and $1000 \mathrm{~m}$ on some occasions. It is observed, for each of them, that the greater the height at which the sonic is located, the greater the integral scale in the turbulent flow. Usually, at $32 \mathrm{~m}$, these scales are, on average, greater than those of $13 \mathrm{~m}$ and the latter higher than at $5.8 \mathrm{~m}$ height. 

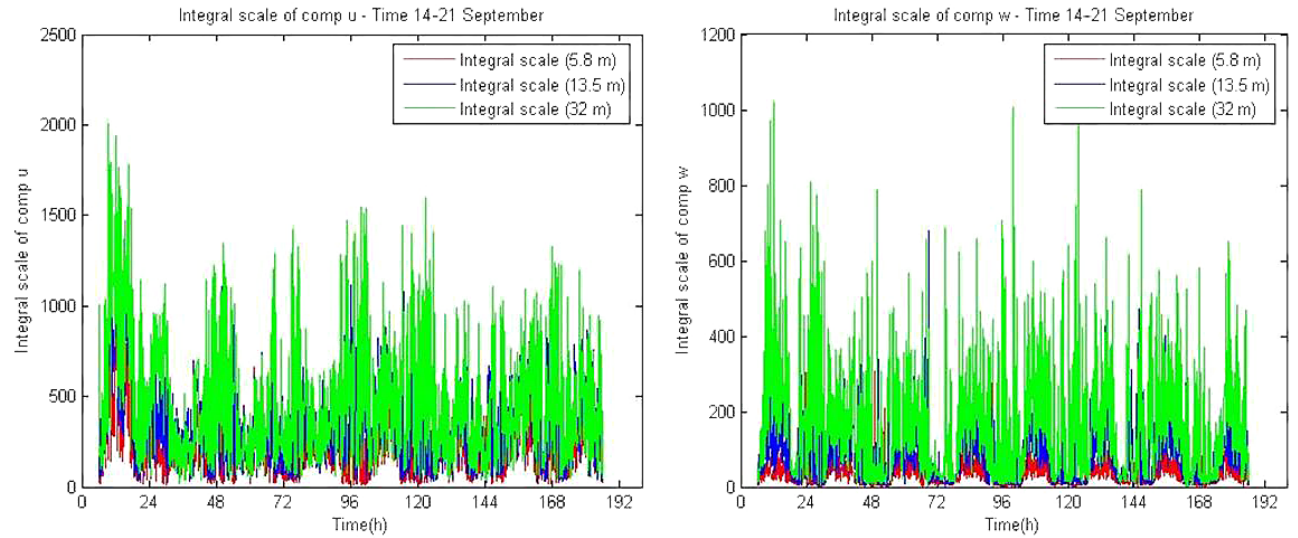

Figure 4. Variation of the integral length scale of horizontal and vertical components vs. time at the three heights.

(a)

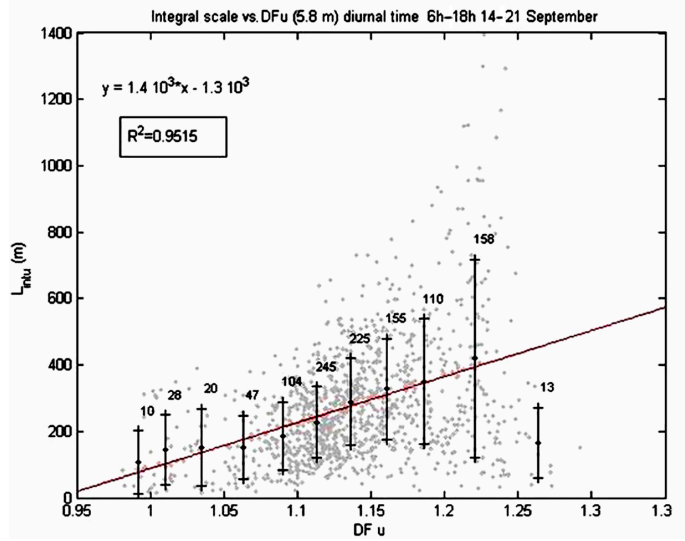

(b)

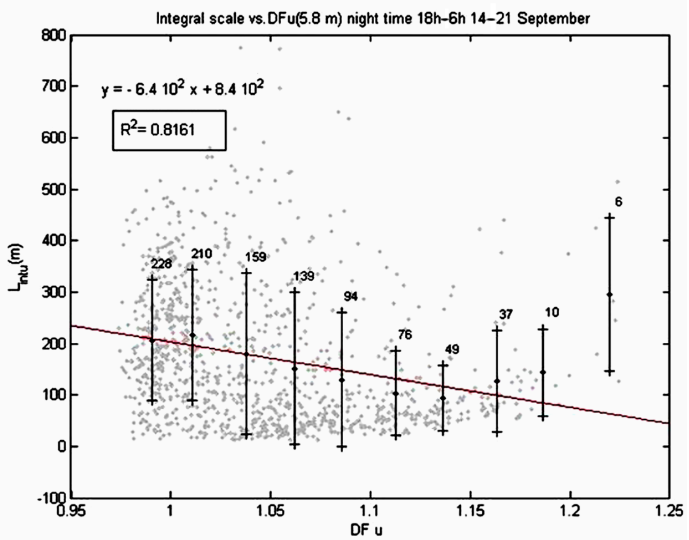

Figure 5. Variations of the integral scale vs. the fractal dimension of the $u^{\prime}$ component of the wind velocity at 5.8 $\mathrm{m}$. (a) Diurnal hours 06:0018:00; (b) night hours 18:00-06:00. On the top left of each graph is indicated the linear regression of the average values $\left(\right.$ a) $L_{\text {intu }}(5.8 \mathrm{~m})=$ $1.4 \times 10^{3} \mathrm{DF}_{u}(5.8 \mathrm{~m})-1.3 \times 10^{3}$ and $(\mathbf{b}) L_{\text {intu }}(5.8 \mathrm{~m})=-6.4 \times 10^{2} \mathrm{DF}_{u}(5.8 \mathrm{~m})+8.4 \times 10^{2}, L_{\text {intu }}$ and $\mathrm{DF}_{u}$ being the integral scale and fractal dimension for the $u$ component, respectively.

Although the conceptual model of turbulence as eddies of various sizes is useful, it is difficult to obtain a correlation between the integral scale and fractal dimension in the atmosphere if we consider values throughout the whole day. However, it is much easier to find a relationship between the integral scale and fractal dimension of horizontal and vertical components of the wind velocity if we separate the hours of the day and night, and hence analyze the influence of the diurnal and night cycles on these parameters. Daylight hours are from 06:00 to 18:00 UTC and in the night from 18:00 to 06:00 UTC. These data sets are analyzed at the three studied heights. Figure 5 shows the variations of the integral scale vs. fractal dimension at the level of $5.8 \mathrm{~m}$ for the horizontal component. As can be appreciated in Fig. 5, in the diurnal hours the average values of the integral scale vs. the fractal dimension can be adjusted to the straight regression line given by the linear equation that appears on the top left of the graph. During those hours these values of the integral scale increase from a few tens of meters to $400 \mathrm{~m}$, with increasing values of the fractal dimension to 1.25 . During the nights the average values of the integral scale decrease with the increase in the fractal dimension. These values also fit a straight regression line as is indicated in Fig. 5. One of the possible explanations for this behavior is that during the diurnal hours the average values of the integral scale increase due to the unstable stratification. During the nights, the existence of the stable stratification decreases the integral scale, with an increase in fractal dimension to the approximate value of 1.2. This tendency also appears at the other two heights, at $13 \mathrm{~m}$ and at $32 \mathrm{~m}$ as shown in Fig. 6. Although during the diurnal cycle at $32 \mathrm{~m}$ the linear fit is not so evident, the maximum scales are in the 1.15-1.20 range of the fractal dimension, as is illustrated in Fig. 6c.

For the vertical component in the three studied levels, the behavior is slightly different. The average values have fit a quadratic function as is indicated in Fig. 7. During the diurnal 
(a)

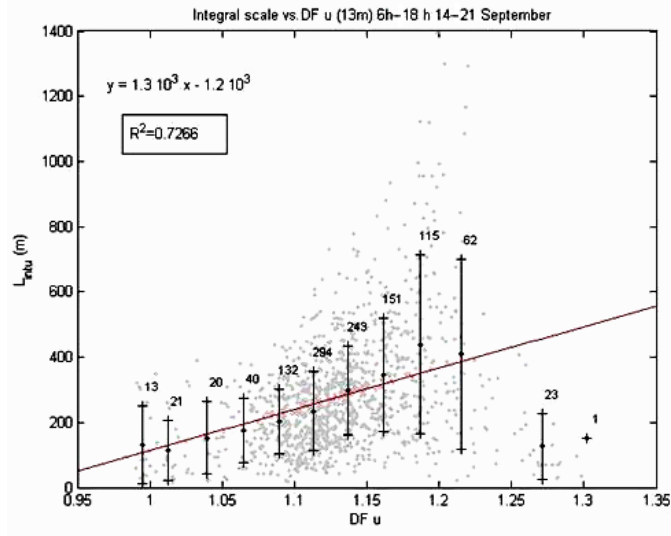

(c)

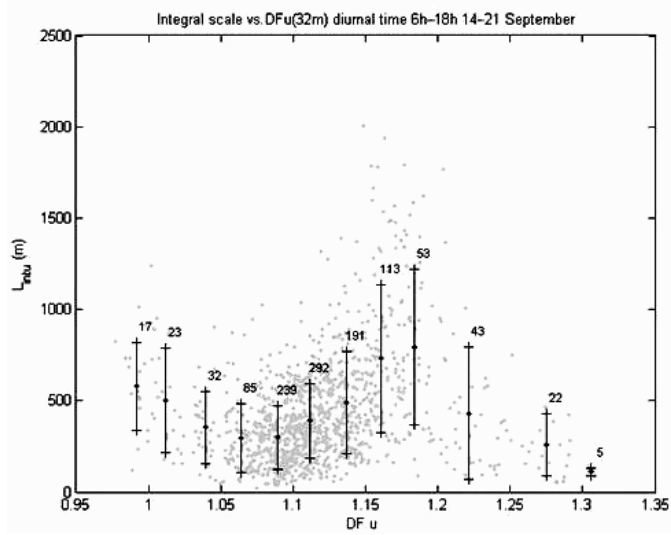

(b)

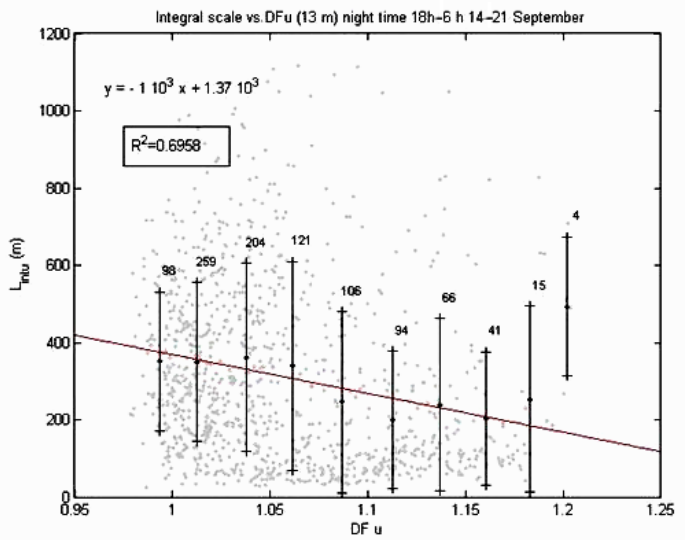

(d)

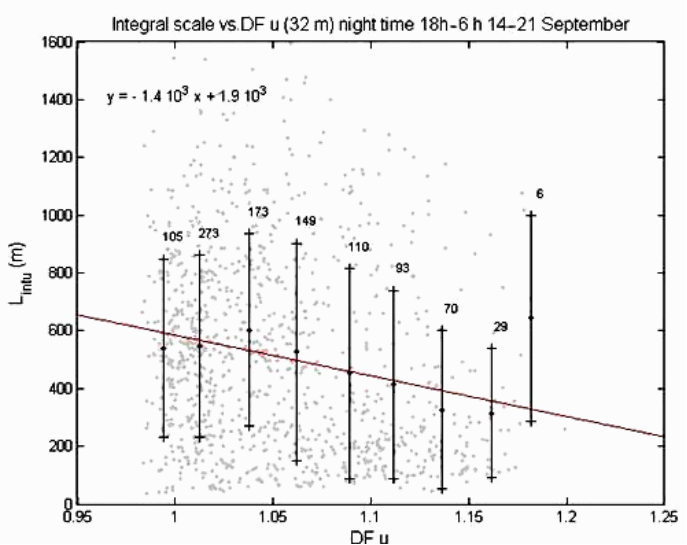

Figure 6. Variations of the integral scale vs. the fractal dimension of the $u^{\prime}$ component at 13 and $32 \mathrm{~m}$. (a) and (c) diurnal hours, (b) and (d) night hours. In the same manner as in Fig. 5, the linear fits are (a) $L_{\text {intu }}(13 \mathrm{~m})=1.3 \times 10^{3} \mathrm{DF}_{u}(13 \mathrm{~m})-1.2 \times$ $10^{3}$, (b) $L_{\text {intu }}(13 \mathrm{~m})=-1 \times 10^{3} \mathrm{DF}_{u}(13 \mathrm{~m})+1.37 \times 10^{3}$, and (d) $L_{\text {intu }}(32 \mathrm{~m})=-1.4 \times 10^{3} \mathrm{DF}_{u}(32 \mathrm{~m})+1.9 \times 10^{3}$.

hours the average values of the integral scale reach maximum scales around the value of the fractal dimension of 1.15 at the three heights. From this value the integral scale decreases when fractal dimension increases. These maximum integral scales depend on the height. At the level of $5.8 \mathrm{~m}$ their sizes reach $50 \mathrm{~m}$ on average and the scattering of the values shows higher values that could reach $100 \mathrm{~m}$. At the level of $13 \mathrm{~m}$ the values are about $100 \mathrm{~m}$, the dispersion of these scales can reach sizes of $200 \mathrm{~m}$ and at the height of $32 \mathrm{~m}$ their larger average scales are approximately around $200 \mathrm{~m}$, and due to the variances of the data set could reach sizes of $400 \mathrm{~m}$. From the value of the fractal dimension value of 1.15 , the scales decrease to a few meters.

Throughout the night the average values of the integral scales decrease with the increase in the fractal dimension in a parabolic way, as is indicated in Fig. 7. This happens due to the stable stratification that occurs at night. This behavior during the diurnal and night hours for the $w^{\prime}$ component of the integral scale is similar to the results obtained for the $u^{\prime}$ component, although the fits of average values are parabolic and not linear. In all these cases our $R^{2}$ values and confidence levels are high, as is indicated in Fig. 7.

\subsection{Relationship between the integral scale and bulk Richardson number}

Among the numerous parameters existing to characterize the degree of stratification in the atmosphere, we will use the bulk Richardson number. The interpretation of this number has already been mentioned in the previous section. Here, we analyze how the integral scale of each one of the $u^{\prime}$ horizontal and $w^{\prime}$ vertical components varies with the bulk Richardson number in diurnal and night cycles in the studied period. These results are shown in Fig. 8 for horizontal components and in Fig. 9 for vertical components. During the daylight hours appear the three kinds of stratification: unstable, neutral and stable, as is shown in the three graphs on the left side of Figs. 8 and 9, each one corresponding to the different heights. In the unstable and neutral stratifications, the integral scales are higher than the integral scales under the influence of the stable stratification. At $5.8 \mathrm{~m}$ for the horizon- 
(a)

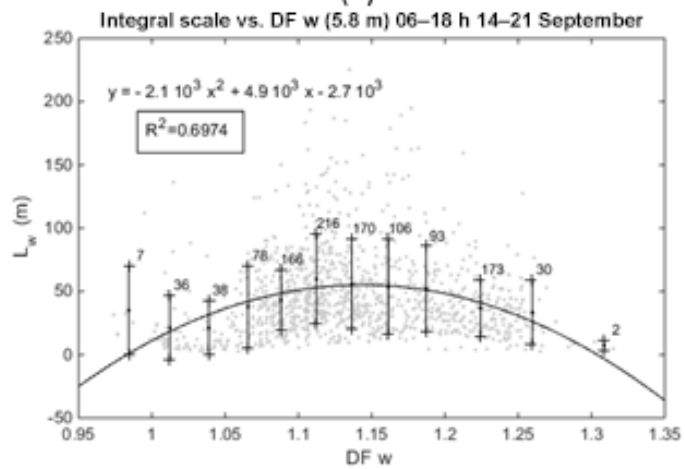

(c)

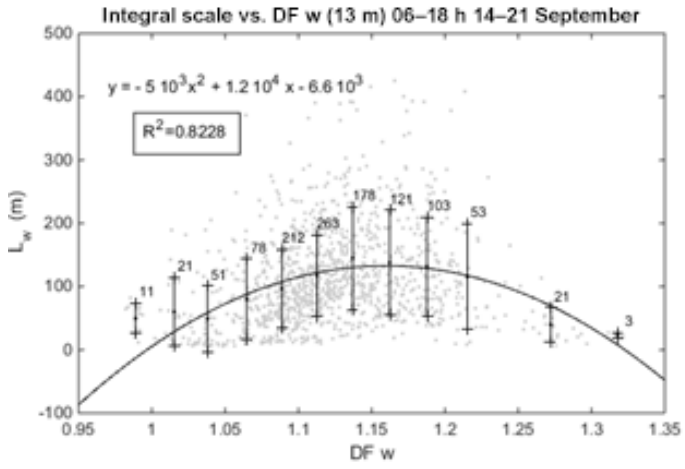

(e)

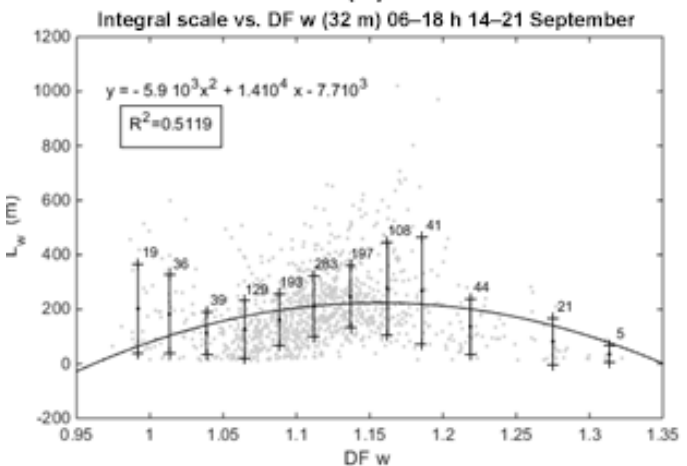

(b)

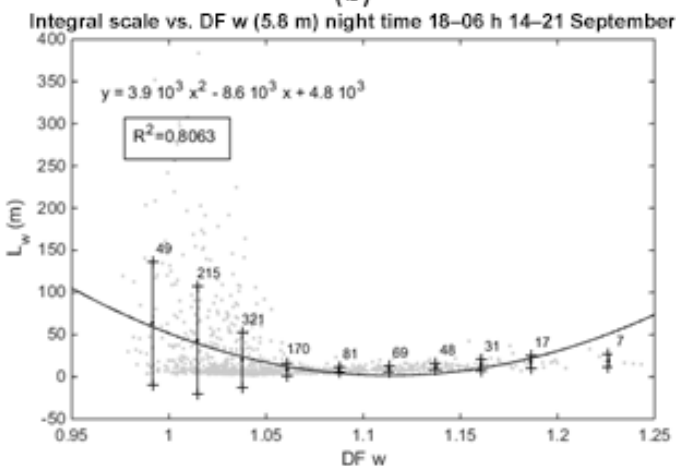

(d)

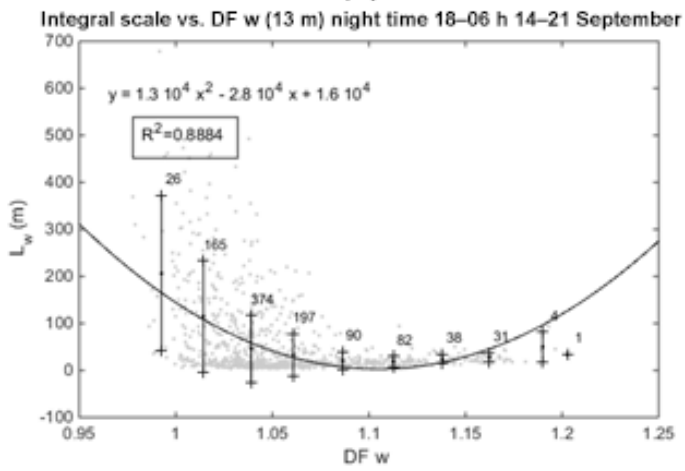

(f)

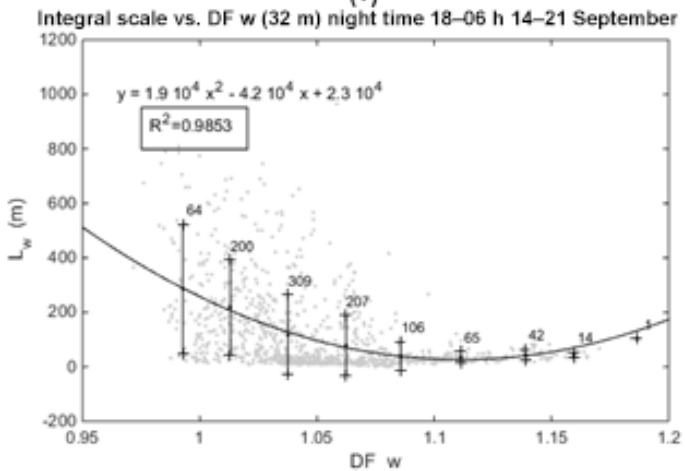

Figure 7. Variations of the integral scale vs. the fractal dimension of the $w^{\prime}$ component at 5.8, 13 and $32 \mathrm{~m}$. (a), (c) and (e) diurnal hours; (b), (d) and (f) night hours. The fits to a quadratic function of the average values appear on the top left of each graph: $y$ variable $L_{\text {intw }}$ and $x$ variable $\mathrm{DF}_{w}$.

tal component these scales vary between $200 \mathrm{~m}$ and values slightly higher than $400 \mathrm{~m}$, and in the case of neutral stratification could increase to $600 \mathrm{~m}$. This same behavior occurs at the other two studied heights, 13 and $32 \mathrm{~m}$, although their scales are slightly higher as illustrated in Fig. 8. During the nights the biggest stability due to positive values of the bulk Richardson number is observed.

The same results are obtained for the integral scales of the vertical component, although their sizes are smaller. At $5.8 \mathrm{~m}$ during the diurnal hours the average values reach about $50 \mathrm{~m}$ and during the night hours their values are below $50 \mathrm{~m}$. At 13 and $32 \mathrm{~m}$ in the diurnal hours the average values could reach about 150 and $200 \mathrm{~m}$ and at the night hours are below 100 and $200 \mathrm{~m}$, respectively.

\subsection{Analysis of the anisotropy with the integral scale}

In the last section we study the relationship between the integral scales of the horizontal and vertical components at different heights: 5.8, 13 and $32 \mathrm{~m}$. In Fig. 10 we represent the integral scale of the $u^{\prime}$ component vs. the integral scale of the $w^{\prime}$ component at three studied heights, and we find linear relations to the average values of these scales. All integral scales measured during the pe- 
(a)

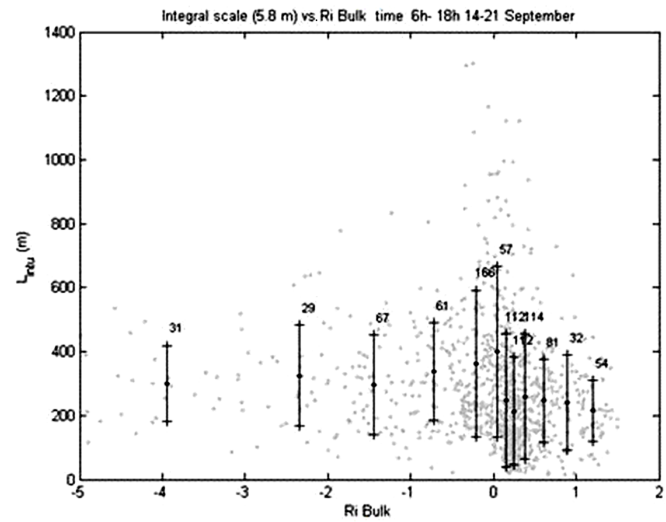

(c)

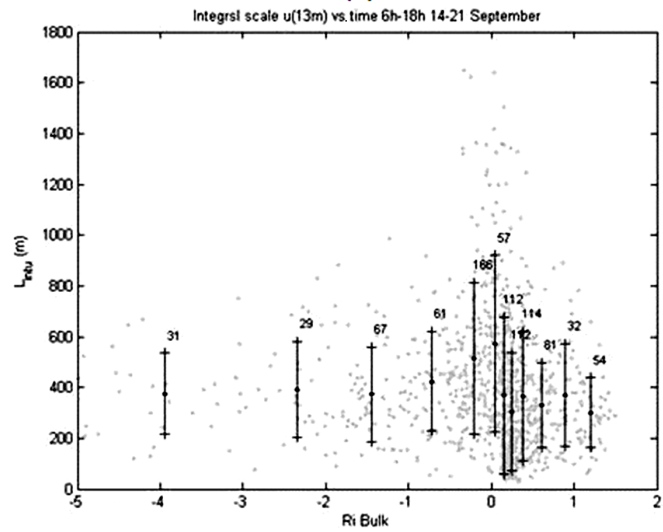

(e)

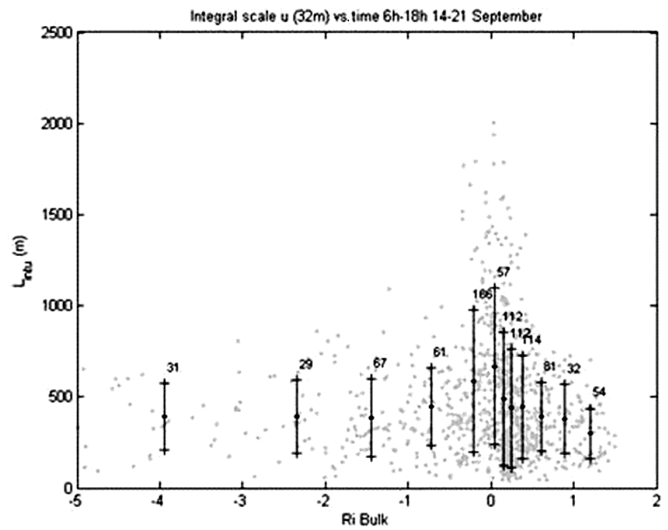

(b)

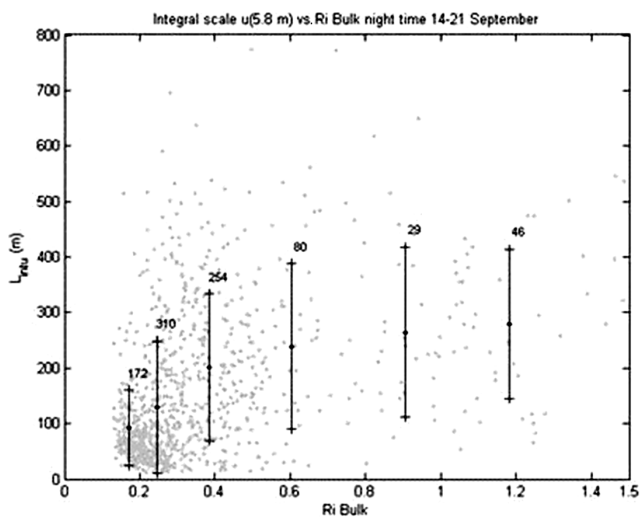

(d)

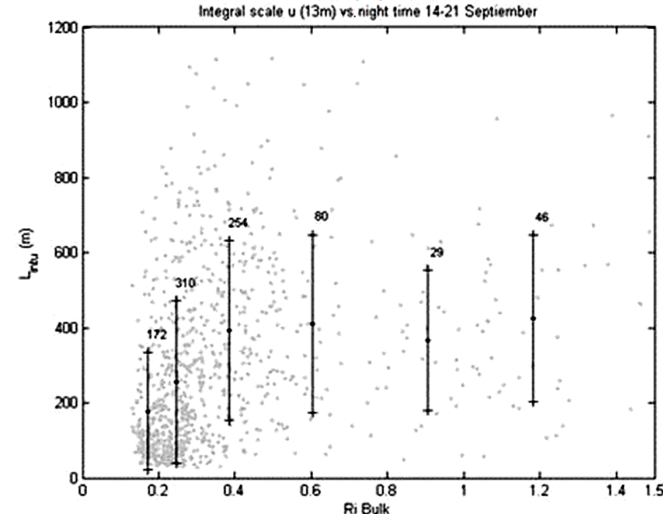

(f)

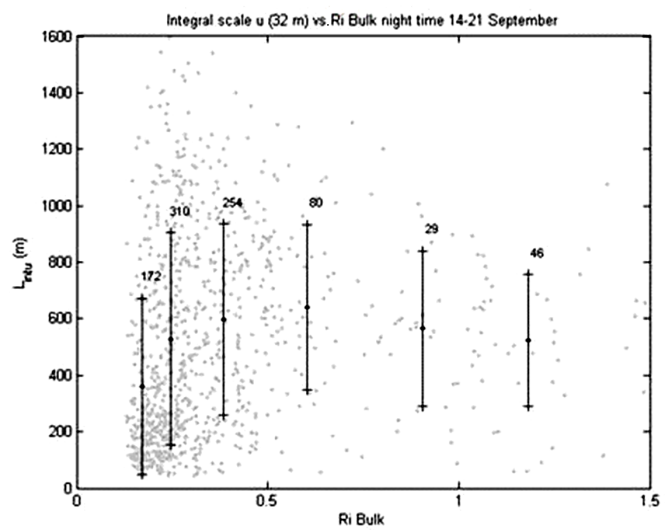

Figure 8. Integral length scales of the $u^{\prime}$ component plotted against the bulk Richardson number at 5.8, 13 and $32 \mathrm{~m}$. (a), (c) and (e) diurnal hours; (b), (d) and (f) night hours.

riod of study from 14 to 21 September appear in this figure. The linear fits obtained are acceptable, with high $R^{2}$ values at 13 and $32 \mathrm{~m}$, as is indicated in Fig. 10 . The linear regression appears on the top left of each graph: at $5.8 \mathrm{~m} L_{\mathrm{intu}}(5.8 \mathrm{~m})=1.46 L_{\mathrm{intw}}(5.8 \mathrm{~m})+178$, at $13 \mathrm{~m} L_{\text {intu }}(13 \mathrm{~m})=0.957 L_{\text {intw }}(13 \mathrm{~m})+275.6$ and at $32 \mathrm{~m}$ $L_{\text {intu }}(32 \mathrm{~m})=0.646 L_{\text {intw }}(32 \mathrm{~m})+370, L_{\text {intu }}$ and $L_{\text {intw }}$ being the average values of the integral scale for the horizontal and vertical components, respectively.

The data in Fig. 10 appear quite scattered and the average values could be representative for finding relationships between these scales. This scatter is due to the large number of uncontrolled variables, nonlocal disturbance, the presence of waves, horizontal inhomogeneity, low-frequency disturbances, etc. These graphs show that the scale measured 
(a)

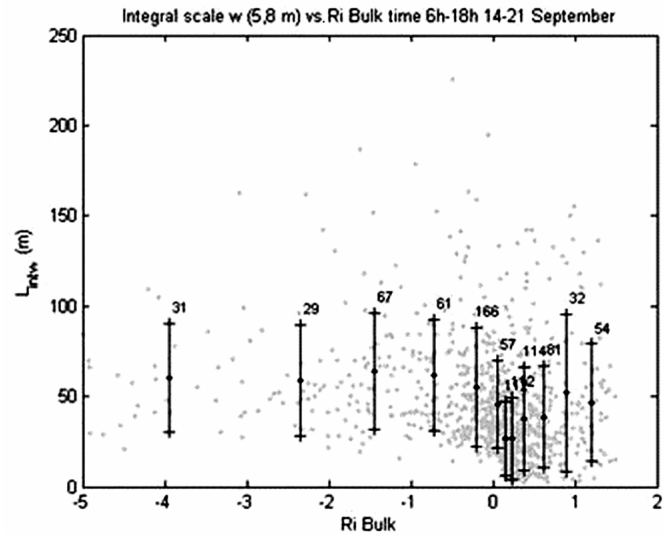

(c)



(e)

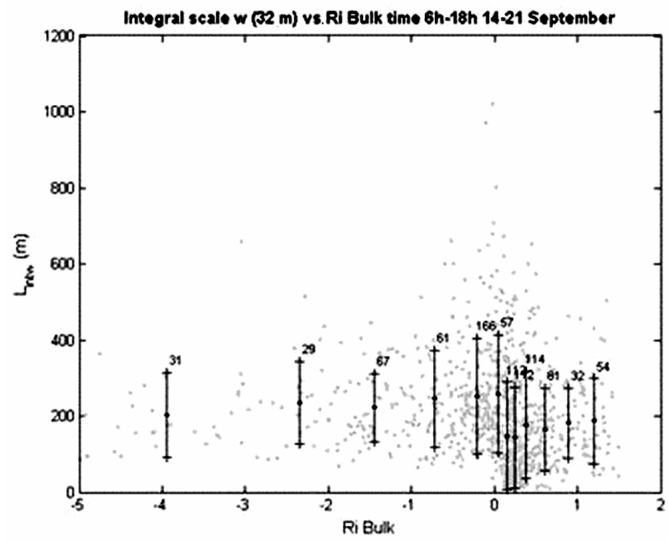

(b)

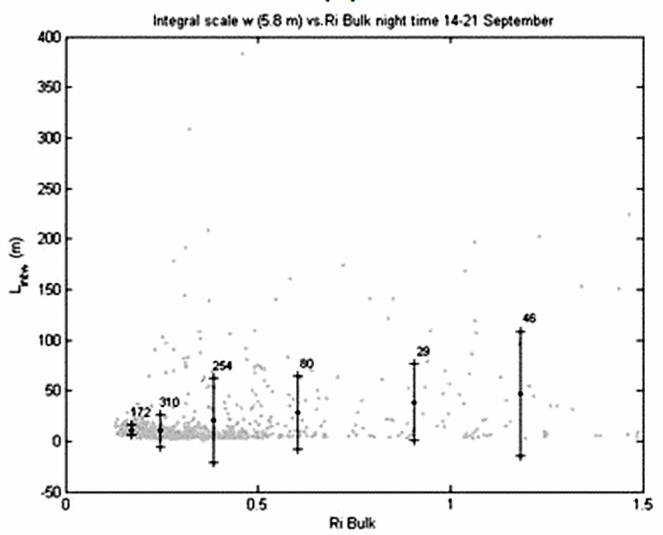

(d)

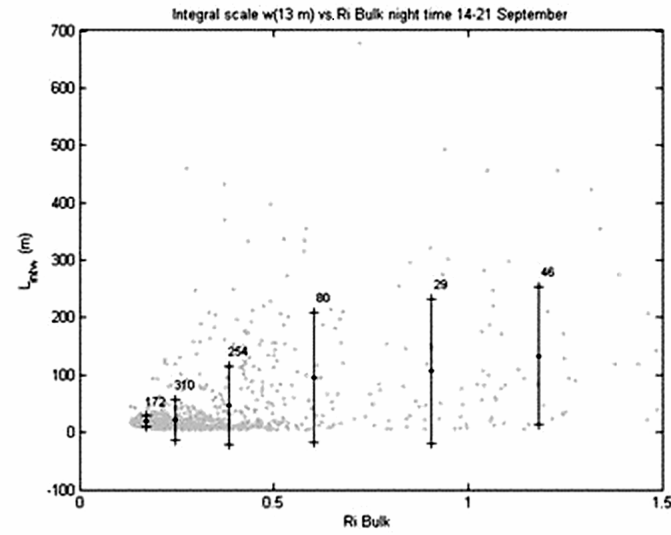

(f)

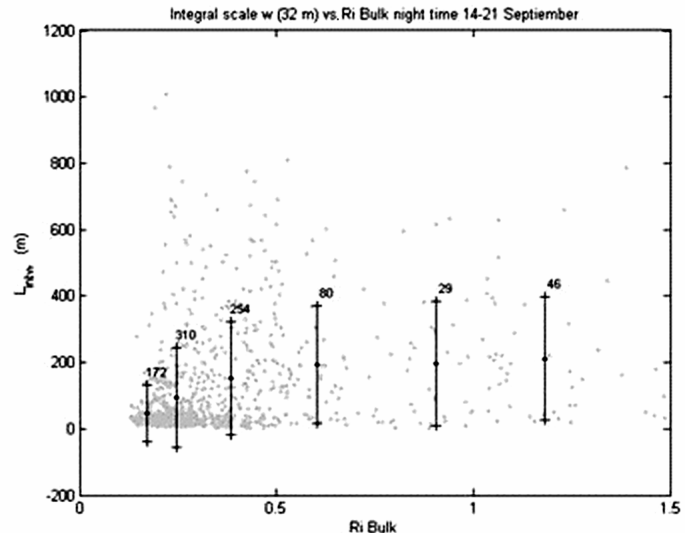

Figure 9. Integral length scales of the $w^{\prime}$ component plotted against the bulk Richardson number at 5.8, 13 and $32 \mathrm{~m}$. (a), (c) and (e) diurnal hours; (b), (d) and (f) night hours.

at $32 \mathrm{~m}$ is nearly always larger than the integral scale measured at $5.8 \mathrm{~m}$. On the basis of the results obtained, we find slight differences between these components; thus, there is anisotropy in atmospheric turbulent flows. In isotropic turbulence the integral scales of both components should be the same at the same height. Only under certain conditions and over limited scales is isotropy a property of turbulence in the stratified atmosphere (Thorpe, 2005). 
(a)

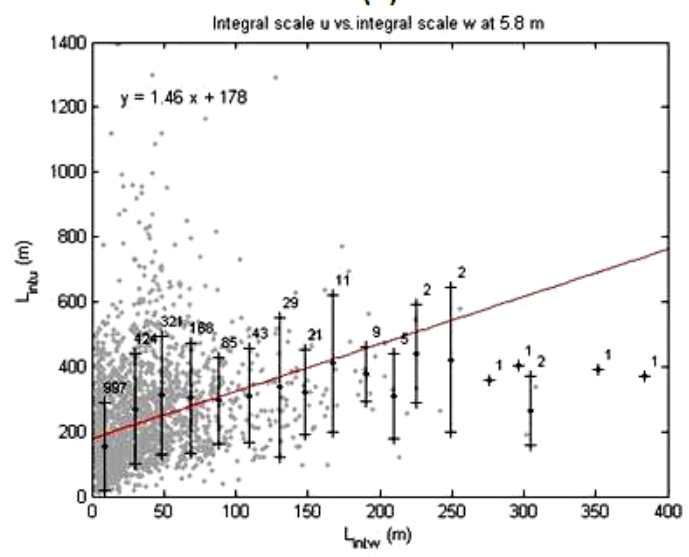

(b)

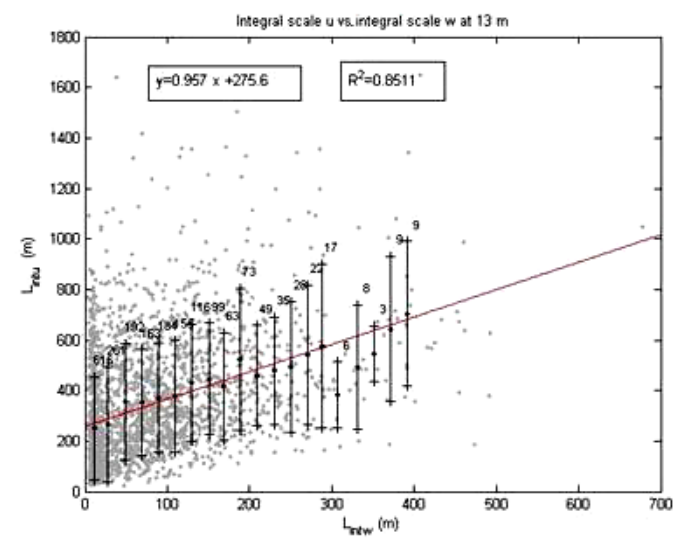

(c)

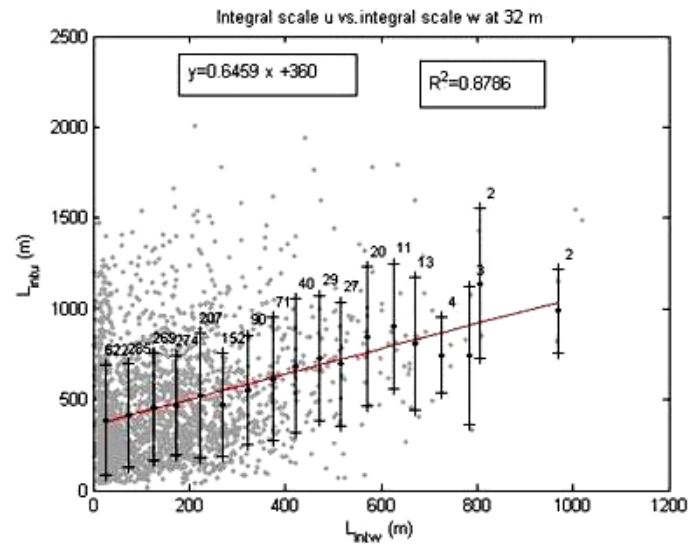

Figure 10. Comparison of integral scales of the $u^{\prime}$ component and $w^{\prime}$ component (a) at $5.8 \mathrm{~m},(\mathbf{b})$ at $13 \mathrm{~m}$ and (c) at $32 \mathrm{~m}$, showing that the average values of theses scales fit to the linear regression indicated on the top left each graph. The data set appears as a cluster around the straight line.

\section{Conclusions}

We have calculated the fractal dimension and the integral scale of the horizontal and vertical components using wind velocity data from sonic anemometers at three different heights: 5.8, 13 and $32 \mathrm{~m}$. The numerical results show slight significant differences in the diurnal and night cycles when the variation of the integral scale is analyzed vs. the fractal dimension. Atmospheric stratification is analyzed for the three heights through the bulk Richardson number, finding the three classical types of stratification along the diurnal cycle. It would be interesting for future works to study the growth of the integral scale in stratified flows and whether this could be due to the inverse cascade on both the diurnal and nighttime cycles. The main conclusions of this study are as follows.

Although all data appear quite scattered in this work, the average values of these magnitudes show interesting results. During the diurnal hours the average values of the integral scale of the horizontal component increase with the increase in fractal dimension to around 1.25 at 5.8 and $13 \mathrm{~m}$ height. At these heights we have found linear fits between these magnitudes with high coefficients of correlation. While at $32 \mathrm{~m}$ the linear fit is not so evident, the maximum scales are in the 1.15-1.20 range of the fractal dimension. One of the possible explanations for this behavior is that during the diurnal hours the average values of the integral scale increase due to the unstable stratification. During the night hours the average values of the integral scale decrease with the increase in the fractal dimension. These values also fit a straight regression line at the three analyzed heights. During the nights the existence of the stable stratification decreases the integral scale, with an increase in the fractal dimension to an approximate value of 1.2 .

For the vertical component of the integral scale the results are similar, even though with slight differences. The average values have fit a quadratic function. During the diurnal hours the average values of the integral scale reach a maximum around the value of the fractal dimension of 1.15 at the three heights. From this value the integral scale decreases with the increase in the fractal dimension to a few meters. 
The different degree of stratification along diurnal hours will be reflected in that different behavior from the value of 1.15. At nights when stability is normally high, the integral scale decreases with increasing fractal dimension in a parabolic way.

In the unstable and neutral stratification the integral scales are higher than the integral scales under the influence of the stable stratification.

To characterize the anisotropy of turbulent flows we have used the comparison of integral scales of horizontal and vertical components, showing that the scale of the $u^{\prime}$ component is almost always larger than the scale of the $w^{\prime}$ component at the same height.

\section{Data availability}

Data are not available form a public data repository (they are raw data with $20 \mathrm{~Hz}$ sampling rate and then huge files). However, all the information about them can be found in the reference Cuxart et al. (2000). On the other hand, original data used in this work can be provided upon request to author.

Acknowledgements. This research has been funded by the Spanish Ministry of Science and Innovation (projects CGL200912797-C03-03). The GR35/10 program (supported by Banco Santander and UCM) has also partially financed this work through the Micrometeorology and Climate Variability research group (no. 910437). Thanks to the participating teams in SABLES 98 for the facilities with the data.

Edited by: J. Rees

Reviewed by: two anonymous referees

\section{References}

Arya, S. P.: Introduction to Micrometeorology, International Geophysics Series, Academic Press, 420 pp., 2001.

Besicovitch, A. S.: On linear sets of points of fractional dimension, Math. Ann., 101, 161-193, 1929.

Cuxart, J., Yagüe, C., Morales, G., Terradellas, E., Orbe, J., Calvo, J., Fernández, A., Soler, M. R., Infante, C., Buenestado, P., Espinalt, A., Joergensen, H. E., Rees, J. M., Vilà, J., Redondo, J. M., Cantalapiedra, I. R., and Conangla, L.: Stable atmospheric boundary layer experiment in Spain (SABLES 98): A report, Bound.-Lay. Meteorol., 96, 337370, doi:10.1023/A:1002609509707, 2000.
Falconer, K. J.: Fractal Geometry Mathematical Foundations and Applications. John Wiley \& Sons, Ltd., 288 pp., 1990.

Frisch, U.: Turbulence, Cambridge Univesity Press, England, 296 pp., 1995.

Grassberger, P. and Procaccia, I.: Characterization of Strange Attractors, Phys. Rev Lett., 50, 346-349, 1983.

Hausdorff, F.: Dimension und äußeres Mass. Mathemastische Annalen., 79, 157-179, 1918.

Kaimal, J. C. and Finnigan, J.: Atmospheric Boundary Layer Flows. Their Structure and Measurement, Oxford University Press, 289 pp., 1994.

Mandelbrot, B. B.: Fractal: Form, Chance and Dimension, Freeman, San Fracisco, 365 pp., 1977.

Mandelbrot, B. B.: The Fractal Geometry of Nature, Freeman, San Fracisco, 497 pp., 1985.

Marino, R., Mininni, P. D., Rosenberg, D., and Pouquet, A.: Largesale anisotropy in stably stratified rotating flows, Phys. Rev. E, 90, 023018, doi:10.1103/PhysRevE.90.023018, 2014.

Monin, A. S. and Yaglom, A. M.: Statistical Fluid Mechanics Mechanics, Vol. 1, Dover Publications, 769 pp., 1971.

Panofsky, H. A. and Dutton, J. A.: Atmospheric Turbulence, Wiley and Sons, 397 pp., 1984.

Peitgen, H., Jürgens, H., and Saupe, D.: Chaos and Fractals, Springer-Verlag, Springer-Science + Business Media, LLC, 971 pp., 2004.

Shirer, H. N., Fosmire, C. J., Wells, R., and Suciu, L.: Estimating the Correlation Dimension of Atmospheric Time Series, J. Atmos. Sci., 54, 211-229, 1997.

Smith, L. and Waleffe, F.: Generation of slow large scales in forced rotating stratified turbulence, J. Fluid Mech., 451, 145-168, 2002.

Stull, R. B.: An introduction to Boundary Layer Meteorology, Kluwer Academic Publishers, Dordrecht, the Netherlands, 670 pp., 1988.

Teunissen, H. W.: Structure of mean winds and turbulence in the planetary boundary layer over rural terrain, Bound.-Lay. Meteorol., 19, 187-221, 1980.

Thorpe, S. A.: The turbulent ocean, Cambridge Universe Press, New York, 439 pp., 2005.

Tijera, M., Cano, J. L., Cano, D., Bolster, B., and Redondo, J. M.: Filtered deterministic waves and analysis of the fractal dimension of the components of the wind velocity, Nuovo Cimento C., 31, 653-667, 2008.

Tijera, M., Maqueda, G., Yaque, C., and Cano, J.: Analysis of fractal dimension of the wind speed and its relations with turbulent and stability parameters, Intech, Fractal Analisis and Chaos in Geosciences, 29-46, 2012.

Vassilicos, J. C. and Hunt, J. C. R.: Fractal dimensions and spectra of interfaces with application to turbulence, Proceedings Mathematical and Physical Sciences, 435, 505-534, 1991. 